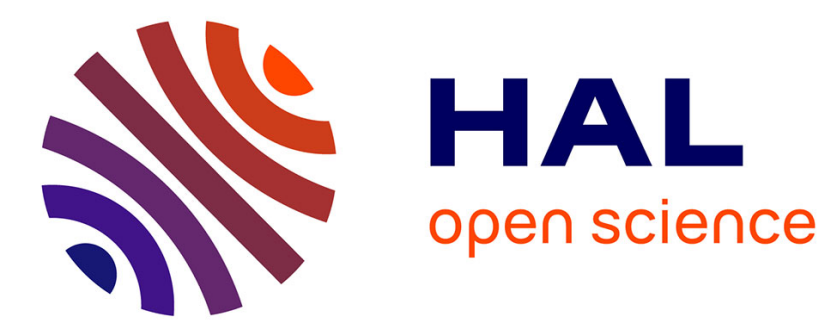

\title{
3D models for vascular lumen segmentation in MRA images and for artery-stenting simulation
}

Leonardo Flórez-Valencia, Johan Montagnat, Maciej Orkisz

\section{To cite this version:}

Leonardo Flórez-Valencia, Johan Montagnat, Maciej Orkisz. 3D models for vascular lumen segmentation in MRA images and for artery-stenting simulation. Ingénierie et Recherche Biomédicale (ITBMRBM), 2007, 28 (2), pp.65-71. 10.1016/j.rbmret.2007.04.001 . hal-00683451

\section{HAL Id: hal-00683451 https://hal.science/hal-00683451}

Submitted on 29 Mar 2012

HAL is a multi-disciplinary open access archive for the deposit and dissemination of scientific research documents, whether they are published or not. The documents may come from teaching and research institutions in France or abroad, or from public or private research centers.
L'archive ouverte pluridisciplinaire HAL, est destinée au dépôt et à la diffusion de documents scientifiques de niveau recherche, publiés ou non, émanant des établissements d'enseignement et de recherche français ou étrangers, des laboratoires publics ou privés. 


\title{
3D models for vascular lumen segmentation in MRA images and for artery-stenting simulation
}

\author{
Leonardo Flórez-Valencia ${ }^{a, b}$, Johan Montagnat ${ }^{a}$, Maciej Orkisz ${ }^{a}$ \\ \{florez, montagnat, orkisz\}@creatis.insa-lyon.fr
}

\begin{abstract}
Arterial stenoses and aneurysms are increasingly treated using stents. The goal of this work is to facilitate the pre-operative choice of the stent's length and diameter. Two models are used to accomplish this task: a deformable cylindrical simplex (DCS) model of the arterial intra-luminal wall and a right generalized cylinder (RGC) model representing the stent's geometrical properties. An angiographic 3D image is first segmented using the DCS model to create a patient-specific vascular model. The RGC model of a folded stent is then slid along the vessel centerline until an interactively chosen delivery location, and the model's geometry is modified to simulate the unfolding of the stent. Lastly, the DCS model is re-meshed to fit the shape of the unfolded-stent model and thus to simulate the shape of the arterial lumen boundaries after stenting. Accuracy of the segmentation was quantitatively evaluated in images of arterial phantoms with stenoses ranging from $50 \%$ to $95 \%$. Mean error of the resulting diameter estimation was $4.24 \%$. The simulation of the stent insertion is qualitatively illustrated using data of a patient with an aneurysm in the aorta arch.
\end{abstract}

Index Terms-Image segmentation, vascular image, deformable model, stenosis quantification, stent, endo-prosthesis.

\section{INTRODUCTION}

\section{A. Medical context}

Diseases of the arterial wall are one of the main causes of death in western countries. A local weakening of the arterial wall leads to a distension, called aneurysm, the rupture of which can cause hemorrhage and stroke. A development of an atherosclerotic plaque (accumulation of lipidic, calcified and fibrous components) leads to a stenosis, i.e. a local stricture of the arterial lumen, which can cause hypoperfusion, ischemia and infarct of the organs irrigated by the affected artery. Increasingly, patients presenting these pathologies undergo an implantation of an endovascular prosthesis or stent [1]. A stent is a tiny metal grid inserted in an artery after an angioplasty procedure has cleared the fatty debris that blocked the blood vessel. It acts as a scaffold to help keep the artery open after removal of the blockage. The device is initially folded. It is inserted into the artery through an incision, using a catheter. Then it is deployed within the diseased region, using either a balloon inflation or the shape-memory effect. Similar to the stents, but covered with a blood-proof tissue, an endo-prosthesis ${ }^{1}$ is placed in an aneurysm to

\footnotetext{
${ }^{a}$ Creatis, CNRS UMR \#5515, Inserm U 630, Lyon, France

${ }^{b}$ Universidad de los Andes, Facultad de Ingeniería, Santafé de Bogotá, Colombia

${ }^{1}$ From here forth "stent" will denote both simple grids and tissue-covered endo-prostheses.
}

canalize the blood flow and reduce the pressure on the arterial wall, thus preventing rupture. The endo-vascular stent grafting provides a less invasive alternative to surgery. However, an appropriate pre-operative choice of the stent's dimensions (length, diameters at the attachment sites, location of the points of articulation) is necessary to avoid complications such as leakage, loosening, artery dissection or obstruction of arterial branches. Precise measurements of lengths, diameters and angulations of the proximal and distal attachment sites are particularly required in the case of the aortic aneurysm stenting. The usual measurement procedure, using a calibrated catheter and Digital Subtracted Angiography, is lengthy and invasive. Furthermore, this technique tends to inaccurately estimate the length of tortuous vessels because the catheter path does not follow the vessel centerline [2]. Non-invasive measurements based on centerline extraction from 3D medical images, namely from magnetic resonance angiography (MRA) and from computed tomography angiography (CTA), provide an efficient alternative to the use of a catheter [3].

The goal of our work is twofold: 1) to construct, from 3D medical images, patient-specific geometrical models of vascular segments and to deduce from them the measurements useful in pre-operative stent-graft planning, 2) to provide a simulation tool able of representing the stent-unfolding process within a given patient's artery, so that clinicians can visually assess the consequences of various choices of the stent's dimensions.

\section{B. Previous works}

Several recent studies aimed at extracting the patients' individual vascular 3D geometry (see [4] for a review) and/or at simulating vascular interventions. The predictive-medicine paradigm was proposed by researchers from the Stanford University. Its goal is a pre-operative assessment of the outcome of various therapeutic strategies. These researchers have carried out a vast project bringing together various software tools in increasingly integrated computational frameworks: SVVL (Stanford Virtual Vascular Laboratory), ASPIRE [5], [6] and Geodesic [7]. In the latter, these tools range from the level-sets based automatic and semi-automatic image segmentation, through the generation of meshes representing both the segmented vessels and the virtual prostheses, until the computational fluid-dynamics (CFD) simulation.

A patient-specific carotid artery model construction and the CFD simulation were carried out in another project [8], in which the vascular geometry was segmented using a tubular 
deformable mesh model [9]. To simulate the artery stenting, this mesh was merged with a cylindrical mesh model of the stent.

In the MedIS-VR project, a virtual endoscopic viewing was used for the pre-operative aortic stent planning. The vascular surface was extracted by an interactive thresholding associated with an advanced Marching Cubes algorithm. The vessel centerline was then automatically deduced, and a virtual stent was placed along this path. Its length and its diameters were finally interactively adjusted [10].

A similar approach of the vascular surface extraction was recently used in [11] where an isosurface is first extracted, based on an interactive thresholding and on the Marching Cubes algorithm, then this surface is deformed towards the local maxima of the intensity gradient. This model however does not include the vessel centerline.

Let us also mention the ViVa (Virtual Vascular) project [12], [13] that concentrated on the simulation of the insertion of surgical needles and of catheters.

To construct the patient-specific model of the vessels, we propose an original 3D cylindrical deformable model. This approach overcomes the limitations of most existing methods (including [7]) based on 2D boundary extraction carried out independently in cross-sectional images along the vessel. Our method differs from [9] and [11] mainly by three characteristics: 1) it includes an automatic initialization of the vessel's centerline and of its approximate local diameters, 2) the model consists of two interacting components, a cylindrical surface and a centerline, hence no post-processing of the surface is necessary to deduce the final centerline, and 3) it is based on a different formalism: the simplex meshes [14]. Indeed, to model the internal wall of the vessel we use a cylindrical simplex surface mesh. A deformable centerline (axis) is attached to this surface in order to control its deformation process. To interactively manipulate the virtual stent, we use a right generalized cylinder (RGC) model that can be compared with the movable discs used in [10].

The entire process can be summarized as follows. First, a $3 \mathrm{D}$ image of the vascular lumen is acquired using the contrast-enhanced magnetic resonance angiography (MRA) technique [15]. Second, the Maracas software, developed in our laboratory [16], is used to choose the vascular segment of interest, to automatically extract a series of points that constitute its centerline (axis) and to estimate the associated local radii. Third, the actual segmentation of the vascular lumen is carried out using the deformable cylindrical simplex (DCS) model, initialized with the previously extracted axis and with the estimated local radii. Then the user interactively introduces into the segmented vessel a RGC model of the folded stent, and slides it along the vessel axis up to the desired location, where the stent is automatically deployed. Lastly, the mesh model of the vessel surface is modified in order to fit the stent's surface.

The construction of the patient-specific vascular models, as well as its evaluation on a set of 3D images, are described in section II. Section III describes the method for artery stentgraft simulation and shows its results.

\section{Segmentation}

\section{A. Initial axis extraction and radius estimation}

Like most deformable models, the DCS model requires an initialization close to the final solution. In particular, the initial axis should fall within the vessel lumen. This initialization can be based on a simple thresholding followed by a skeletonization step providing the medial axis of the binary structure. Many other algorithms have been reported in the literature, which extract the centerline of the vessels and (some of them) estimate their local radii from 3D medical images [17]-[27]. In our current implementation, the axis is extracted using a very efficient extensible skeleton method that we described in detail in an earlier work [28]. This method is based on a tracking strategy that begins with a given seed (3D point) and, iteratively, estimates the subsequent axis points within a two-step procedure: first, the new point position is predicted; then this position is corrected under the influence of internal and external forces. The internal forces control the elasticity and the flexibility of the axis, while the external forces attract its points towards central locations within the vessel. The external forces are deduced from image moments computed within a spherical neighborhood of variable size. The radius estimation is also based on this multi-scale analysis of the image moments. It consists in detecting the largest scale at which the eigenvalues of the inertia matrix remain approximately equal.

The coarse representation of the vessel obtained at this step can be written as an ordered set $\mathcal{C}_{v}$ of $N$ axis points $\mathbf{a}_{i}$ and of their associated radii $r_{i}$ :

$$
\mathcal{C}_{v}=\left\{\left(\mathbf{a}_{i}, r_{i}\right) \in \mathbb{R}^{3} \times \mathbb{R}^{+}\right\}, 0 \leq i<N .
$$

\section{B. Deformable cylindrical simplex model of the vessel}

Both the centerline and the surface of the vessel can be represented using simplex meshes. The $n$-dimensional $k$-simplex meshes [14] are discrete mesh representations characterized by their regular topology that simplifies computations involved in the deformation process. Each $n$-dimensional vertex has exactly $k+1$ neighbor vertices. To represent the surface of the vessel we use a 3-dimensional 2-simplex mesh, while a 3-dimensional 1-simplex mesh is used to represent the vessel centerline.

1) Initial model generation: As the model described by equation 1 implicitly corresponds to the 1-simplex definition of the vessel axis, we complete this initialization by calculating a cylindrical surface attached to this axis, having local estimated radii $r_{i}$. A set of $M$ vertices $\mathbf{v}_{j}$ is calculated to complete the mesh model $\mathcal{M}_{v}$. The complete DCS model is the union of the centerline $\mathcal{C}_{v}$ and of a cylindrical surface mesh $\mathcal{S}_{v}=\left\{\mathbf{v}_{j} \in \mathbb{R}^{3}, 0 \leq j<M\right\}$. It also includes the spatial relationships between the vertices, as well as the constraints that will be detailed hereafter (see section II-B.2).

2) DCS model evolution: According to the classical deformable models framework [29], the mesh deformation is controlled by an internal (regularizing) energy term and by an external (data-driven) term. The discretization of the secondorder energy-minimization scheme, for each simplex vertex, 
leads to the evolution equation:

$$
\mathbf{v}_{j}^{t+1}=\mathbf{v}_{j}^{t}+\gamma\left(\mathbf{v}_{j}^{t}-\mathbf{v}_{j}^{t-1}\right)+\mathbf{f}^{\mathrm{int}}\left(\mathbf{v}_{j}^{t}\right)+\beta \mathbf{f}^{\mathrm{ext}}\left(\mathbf{v}_{j}^{t}\right),
$$

where $\mathbf{v}_{j}^{t}$ denotes the location of the vertex $\mathbf{v}_{j}$ at the iteration $t$, $\beta \in[0,1]$ is the weight of the external force and $\gamma$ is a damping parameter. In the sequel, the notations will be simplified by omitting the superscript $t$ where this does not lead to ambiguity. The computation of the internal force $\mathbf{f}^{\text {int }}\left(\mathbf{v}_{j}\right)$ is specific to the mesh geometry. It uses a criterion enforcing the regularity of the surface curvature. The simplex mesh representation permits a definition of the position of a vertex relatively to its neighbors and of discrete parameters, such as the local curvature, which is convenient for the computation of the surface regularization (see [14], [30] for details). The external force $\mathbf{f}^{\text {ext }}\left(\mathbf{v}_{j}\right)$ attracts the model towards the vessel boundary in the image (see section II-B.3 for details).

Equation 2 defines the local deformation of each surface vertex but it does not take into account the particular global cylindrical shape and the expected properties of a vessel. To improve its deformation behavior, the surface is bound to its centerline: each surface vertex $\mathbf{v}_{j}$ is associated with the 3 closest centerline vertices $\left\{\mathbf{a}_{i-1}, \mathbf{a}_{i}, \mathbf{a}_{i+1}\right\}$. In consequence, each centerline vertex $\mathbf{a}_{i}$ is bound to a set of surface vertices denoted $\mathcal{E}_{i}$. Each pair of vertices $\left(\mathbf{a}_{i}, \mathbf{v}_{j}\right)$ is weighted by a coefficient $\nu_{i j}$ proportional to the distance between $\mathbf{a}_{i}$ and $\mathbf{v}_{j}$, and such that $\sum_{\mathbf{v}_{j} \in \mathcal{E}_{i}} \nu_{i j}=1$. When the surface undergoes a deformation, the axis bends accordingly through an external force resulting from the surface forces reported onto the axis:

$$
\mathbf{f}^{\mathrm{ext}}\left(\mathbf{a}_{i}\right)=\sum_{\mathbf{v}_{j} \in \mathcal{E}_{i}} \nu_{i j} \mathbf{f}^{\mathrm{ext}}\left(\mathbf{v}_{j}\right) .
$$

Conversely, the centerline bending is reported onto the surface to ensure a homogeneous deformation behavior of the cylinder surface. It is obtained through an additional external force acting on the surface, which can be decomposed into the sum of an axial component $\mathbf{f}^{\text {axial }}\left(\mathbf{v}_{j}\right)$ (each vertex tends to follow the axis global motion) and of a radial component $\mathbf{f}^{\text {radial }}\left(\mathbf{v}_{j}\right)$ (each vertex tends to align on a circle around the centerline):

$$
\begin{gathered}
\mathbf{f}^{\text {axial }}\left(\mathbf{v}_{j}\right)=\frac{1}{3} \sum_{k=i-1}^{k=i+1} \nu_{k j} \mathbf{f}^{\mathrm{ext}}\left(\mathbf{a}_{k}\right), \\
\mathbf{f}^{\text {radial }}\left(\mathbf{v}_{j}\right)=\mathbf{v}_{j}^{\perp}-\mathbf{v}_{j}+\left((1-\xi)\left\|\mathbf{v}_{j}^{\perp}-\mathbf{v}_{j}\right\|+\xi \overline{r_{j}}\right) \mathbf{n}_{j},
\end{gathered}
$$

where $\mathbf{v}_{j}^{\perp}$ is the orthogonal projection of $\mathbf{v}_{j}$ onto the centerline, $\mathbf{n}_{j}$ is the unit normal vector of the centerline in $\mathbf{v}_{j}^{\perp}$ (i.e. $\mathbf{n}_{j}=\frac{\mathbf{v}_{j}-\mathbf{v}_{j}^{\perp}}{\left\|\mathbf{v}_{j}-\mathbf{v}_{j}^{\perp}\right\|}$ ), $r_{k}$ is the radius in $\mathbf{a}_{k}$ (mean distance to the centerline from the surface vertices $\left.\mathbf{v}_{j} \in \mathcal{E}_{k}\right), \overline{r_{j}}=$ $\frac{1}{3} \sum_{k=i-1}^{k=i+1} r_{k}$ and $\xi$ is a radial weight. When $\xi=1$ the radial force attempts to maintain circular cross-sections, while with $\xi=0$ the circularity constraint is completely relaxed.

The surface vertices are thus subject to the internal and external forces (local forces) plus the axial and radial forces (centerline forces). Let the parameter $\lambda \in[0,1]$ weight the contributions of the local and centerline forces, then the equation 2 becomes:

$$
\begin{aligned}
\mathbf{v}_{j}^{t+1}=\mathbf{v}_{j}^{t}+\gamma\left(\mathbf{v}_{j}^{t}\right. & \left.-\mathbf{v}_{j}^{t-1}\right)+\lambda\left(\mathbf{f}^{\text {int }}\left(\mathbf{v}_{j}^{t}\right)+\beta \mathbf{f}^{\text {ext }}\left(\mathbf{v}_{j}^{t}\right)\right) \\
& +(1-\lambda)\left(\mathbf{f}^{\text {axial }}\left(\mathbf{v}_{j}^{t}\right)+\mathbf{f}^{\text {radial }}\left(\mathbf{v}_{j}^{t}\right)\right),
\end{aligned}
$$

where $\lambda$ controls the cylinder-like behavior of the surface. With $\lambda=1$, the surface deformation is independent of the centerline shape and of the cross-sectional circularity constraint, i.e. equation 5 is identical to equation 2. With $\lambda=0$, the surface bends exactly according to the centerline shape, and the contribution of the cross-sectional circularity constraint is only dependent on $\xi$. With $0<\lambda<1$ one can obtain intermediate behaviors.

The vertices iteratively evolve until obtaining (at a discrete time $t=T_{s}$ ) a stable model denoted $\mathcal{M}_{v}^{T_{s}}$ (see section II-C).

3) External force for MRA images: The external force $\mathrm{f}^{\mathrm{ext}}$ is dependent on the image acquisition modality, and can use either the image gradient or an iso-value of the image intensity. An empirical study on contrast-enhanced MRA images of vascular phantoms with stenoses [31] has shown that the actual boundary does not correspond to the gradient maximum but rather to $45 \%$ of the local intra-luminal maximum of intensity. For a given vertex $\mathbf{v}_{j}$ this maximum is sought within a sphere $\mathcal{B}\left(\mathbf{a}_{i}, r_{i}\right)$ centered at the axis point $\mathbf{a}_{i}$ closest to $\mathbf{v}_{j}$, and having a radius equal to the initially estimated local radius $r_{i}$ of the vessel. The external force $\mathbf{f}^{\operatorname{ext}}\left(\mathbf{v}_{j}\right)$ is defined as a vectorial displacement: $\mathbf{f}^{\operatorname{ext}}\left(\mathbf{v}_{j}\right)=\mathbf{v}_{j}-\mathbf{p}_{j}$. The point $\mathbf{p}_{j}$ is defined as:

$$
\begin{array}{r}
\mathbf{p}_{j}=\underset{\mathbf{q}_{j} \in \mathcal{L}_{j}}{\arg \min }\left|\mathcal{I}\left(\mathbf{v}_{j}\right)-I\left(\mathbf{q}_{j}\right)\right|, \\
\text { with: } \quad \mathcal{I}\left(\mathbf{v}_{j}\right)=0.45 \max _{\mathbf{b}_{i} \in \mathcal{B}\left(\mathbf{a}_{i}, r_{i}\right)}\left[I\left(\mathbf{b}_{i}\right)\right],
\end{array}
$$

where $I(\mathbf{x})$ is the image intensity at coordinate $\mathbf{x}$. Similarly to [14], the search of the point $\mathbf{p}_{j}$ is carried out along a linear path $\mathcal{L}_{j}$ (a list of image voxels $\mathbf{q}_{j}$ ) described by the surface normal $\mathbf{n}_{j}$. The best candidate is the one having the intensity closest to the local isovalue $\mathcal{I}\left(\mathbf{v}_{j}\right)=\mathcal{I}_{i}$.

\section{Evaluation}

The accuracy and the reproducibility of our segmentation method based on the DCS model was evaluated on seven 3D MRA images (figure 1a) of physical phantoms [32]. The phantoms' internal surface represents the arterial intra-luminal shape, each one having circular reference sections (diameter of $6 \mathrm{~mm}$ ) and two stenoses (figure 1b) of variable shape, position, eccentricity and known severity (between $50 \%$ and 95\%). The images were acquired in realistic conditions with in-plane resolution of $0.78 \mathrm{~mm} \times 0.78 \mathrm{~mm}$ and slice thickness between $0.75 \mathrm{~mm}$ and $1 \mathrm{~mm}$.

For each image we obtained a stable segmentation (figure 1d) within a maximum of 40 iterations. However, the actual segmentation time depends on the number of simplex vertices: a model containing 100 vertices converged in $1.80 \mathrm{~s}$ on a PIII $800 \mathrm{MHz}$ personal computer ${ }^{2}$. Then each resulting axis was resampled (100 evenly spaced points) and the local radii were recalculated for each mesh at these points. In the

\footnotetext{
${ }^{2}$ This time value includes an iterative $3 \mathrm{D}$ rendering of the complete mesh after each iteration of the model evolution.
} 

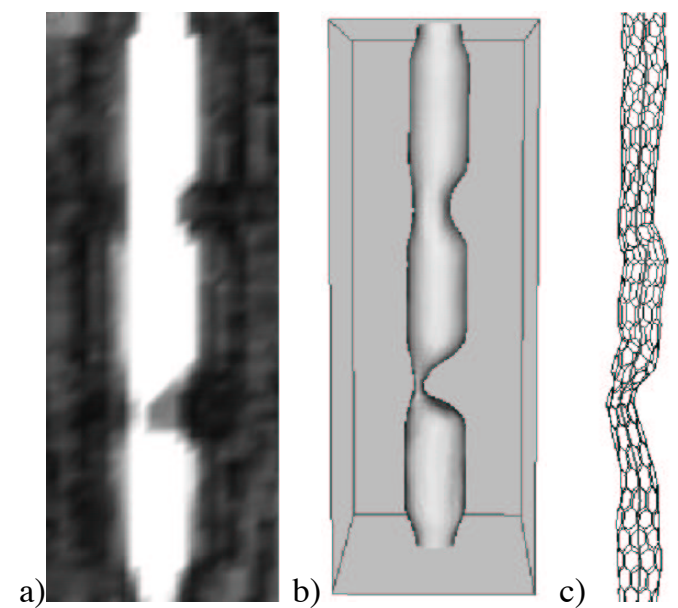

d)

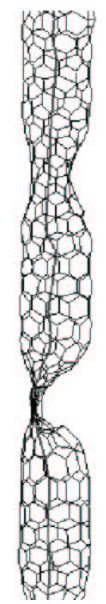

Fig. 1. Vascular phantom and DCS model evolution. From left to right: slice from a 3D MRA image of an arterial phantom with 2 stenoses, shadedsurface rendered true shape of the phantom, mesh initialized according to the coarse model extracted from the MRA image (see section II-B.1), final mesh resulting from the segmentation.

normal sections the average estimated diameter was equal to $6.25 \mathrm{~mm} \pm 0.12 \mathrm{~mm}$. Although the diameter was slightly overestimated $(4.2 \%)$, the errors remain significantly smaller than the voxel size. The standard deviation of $0.12 \mathrm{~mm}(2 \%$ of the reference diameter) gives an idea of the reproducibility, as it corresponds to measurements obtained along the reference sections within different phantoms having the same diameter. The average stenosis-quantification error was equal to $7.14 \%$. This still corresponds to a sub-voxel accuracy of the segmentation.

\section{Stent Simulation}

\section{A. Right generalized cylinder model of the stent}

The stent is modeled as a RGC that can adapt its geometry to a selected zone within the simplex mesh $\mathcal{M}_{v}^{T_{s}}$ representing a vessel. A RGC is defined by its centerline and by a stacking of planar contours locally perpendicular to the centerline, which approximate the cylinder's surface [33]. Similarly to equation 1, we define a $R G C$ stent as:

$$
\mathcal{C}_{s}=\left\{\left(\mathbf{a}_{k}, r_{k}\right) \in \mathbb{R}^{3} \times \mathbb{R}^{+}\right\}, 0 \leq k<N_{s},
$$

where $N_{s}$ is the cardinality of the set $\mathcal{C}_{s}$, i.e. the number of the axis points and of the corresponding planar contours that compose the stent model. The simulation of the vessel stenting is carried out in two steps: (1) fitting the stent centerline to the vessel centerline, and (2) fitting the stent radii to the attachment sites in healthy vascular segments.

1) Placement of a stent in an artery: To make the stent axis and the vessel axis fit, we need a continous representation of both point sets. Let $\left[\begin{array}{lll}a_{k}^{x} & a_{k}^{y} & a_{k}^{z}\end{array}\right]$ represent the 3D coordinates of the $k$-th point of the stent axis. Let $\mathcal{A}_{s}$ be a parametric curve defined by the Catmull-Rom spline equation [34]:

$$
\begin{gathered}
\mathcal{A}_{s}(k, \tau)=0.5\left[\begin{array}{rrrr}
\tau^{3} & \tau^{2} & \tau & 1
\end{array}\right] \mathbf{P A}, \quad \tau \in[0,1], \\
\mathbf{P}=\left[\begin{array}{rrrr}
-1 & 3 & -3 & 1 \\
2 & -5 & 4 & -1 \\
-1 & 0 & 1 & 0 \\
0 & 2 & 0 & 0
\end{array}\right], \quad \mathbf{A}=\left[\begin{array}{llll}
a_{k-1}^{x} & a_{k-1}^{y} & a_{k-1}^{z} \\
a_{k}^{x} & a_{k}^{y} & a_{k}^{z} \\
a_{k+1}^{x} & a_{k+1}^{y} & a_{k+1}^{z} \\
a_{k+2}^{x} & a_{k+2}^{y} & a_{k+2}^{z}
\end{array}\right]
\end{gathered}
$$

To simplify the notations, the parameters $k$ and $\tau$ can be expressed using a new parameter $s_{s} \in[0,1]: \mathcal{A}_{s}\left(s_{s}\right) \equiv$ $\mathcal{A}_{s}\left(k\left(s_{s}\right), \tau\left(s_{s}\right)\right)$. Similarly we define a parametric curve $\mathcal{A}_{v}\left(s_{v}\right)$ representing the vessel centerline.

The user interactively sets a value $s_{v 0}$ for the parameter $s_{v}$ by sliding one end of the folded stent model to the corresponding location in the curve $\mathcal{A}_{v}$. The stent is placed starting from $s_{v 0}$ using a function $\hat{s}_{v}\left(s_{s}\right)$ that maps the stent axis onto the vessel axis (figure $2 b$ ):

$$
\hat{s}_{v}\left(s_{s}\right)=s_{v 0}+s_{s} \frac{l_{s}}{l_{v}},
$$

where $l_{v}$ is the vessel axis length and $l_{s}$ is the stent length.

2) Stent deployment: Once placed, the stent is deployed. We use a simplified geometrical relationship function (figure 2) between its diameter and length, based on the mechanical behavior [35]. There are two strategies of the stentdeployment simulation. The first one corresponds to the balloon-expandable stents. In this case, the radius is userdefined, and the length is directly deduced from the relationship known for the given stent. In the sequel we describe a strategy corresponding to auto-expandable stents.

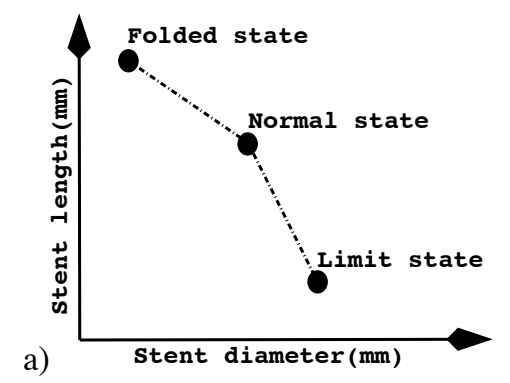

b)

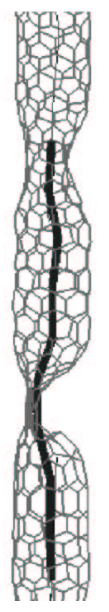

c)

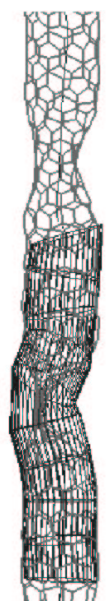

Fig. 2. Stent deployment. (a) Geometrical relationship for a balloonexpandable stent, estimated from the mechanical model described in [35]. This figure shows three state control points: a folded state, a mechanical resistance limit state and a normal state, for which the stent was designed. (b) Simulation of a folded stent placed in a highly stenosed zone. (c) The same stent but deployed.

As the user is supposed to place the end of the stent within a healthy segment of the vessel, it is reasonable to first inflate the stent model until it reaches the radius $r_{v 0}$ corresponding to $s_{v 0}=\hat{s}_{v}(0)$ in the vessel model $\mathcal{M}_{v}^{T_{s}}$. The length $L\left(r_{v 0}\right)$ is deduced from the length/radius relationship known for the 
given stent. Then it may be necessary to adjust the radius at the opposite end of the stent, according to the vessel radius $r_{v 1}$ at $\hat{s}_{v}(1)$. The length $L\left(\left(r_{v 0}+r_{v 1}\right) / 2\right)$ is recalculated and all local radii inside the action zone of the stent are linearly interpolated between $r_{v 0}$ and $r_{v 1}$. In practice this adjustment does not need to be reiterated more than 2 or 3 times.

\section{B. Arterial lumen remodeling}

At this stage of the simulation, the physician can already check whether the dimensions and placement of the stent are appropriately chosen (figure 2c). However, an additional step is necessary if a display of the post-stent vessel lumen surface shape is desired. Indeed, the RGC surface is not regular. Therefore, the artery simplex surface is re-meshed: all vertices inside the deployed stent zone are moved to lay on the surface of the stent. In practice, this procedure is carried out as follows: 1) the DCS centerline remains unchanged and so do the surface vertices not linked with the centerline vertices comprised within the stent action zone: $\left[s_{v 0}, s_{v 1}\right] \equiv$ $\left.\left[\hat{s}_{v}(0), \hat{s}_{v}(1)\right], 2\right)$ the surface is re-meshed within this interval, so that the local radii of the DCS model are equal to the corresponding radii of the RGC model.

\section{Stented arteries in patients}

In images from patients, at this moment, both segmentation and stenting simulation have only been visually evaluated. In general, the simulation is very useful to assess the positioning of the stent and if the stent diameter fits to the vessel's healthy segments (fig. 3). However, the segmentation is sometimes inaccurate when two vessels are too close and cannot be distinguished because of an insufficient resolution of the images. In some cases we also noticed that the vessel axis is not well centered within the vessel lumen. This occurs when the vessel bends strongly. In these cases the deployed stent is not well centered, too. These results can be improved by a careful tuning of the parameters of the model, namely $\lambda$ and $\beta$. Nevertheless a better solution would probably be to add an image force acting directly on the axis and attracting it towards local gravity centers or other medialness-criterion maxima [36].

\section{DISCUSSION}

A right generalized cylinder model was used for simplified simulation of the stent manipulation and deployment within a vessel. This is a reasonable approximation of simple grids and was also used in [10]. To achieve a realistic simulation of aortic endo-prostheses covered with blood-proof tissue, a more complex model is desirable. This model may remain in the RGC family but should have segments of variable length articulated between them. A deformable cylindrical model was used for vessel surface segmentation and representation. This model combines a 3D surface and a centerline linked together. A 3D surface is better suited for these purposes than the RGC model that is implicitly used by many authors. Indeed, when using a 3D-surface model for the purpose of representation, its regularity can be controlled easier than in a RGC model.

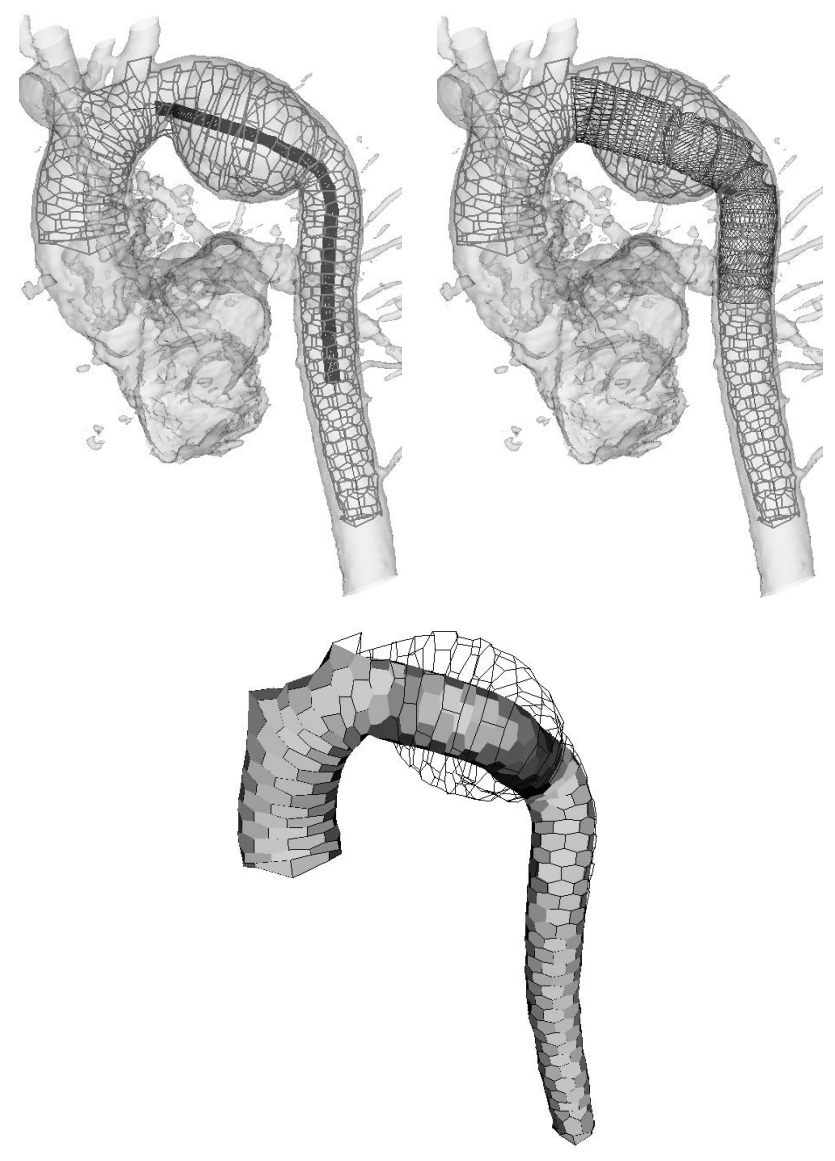

Fig. 3. Simulated stent in a real aneurysm within the aorta arch. Simplex mesh representing the result of the segmentation, with a stent placed folded (left) and deployed (right). Surface rendering of the simplex mesh fitting to the deployed stent (down).

Furthermore, compared with 2D-contour extraction in planes orthogonal to the centerline, segmentation using a 3D-surface model can provide additional shape-constraints that are useful namely in poorly contrasted or noisy regions. The inclusion of the centerline in the DCS model helps managing these constraints in an explicit way. Moreover, the centerline is necessary for the measurements of length and very useful for the visualization purposes: curvilinear multiplanar reformatting, virtual endoscopic flight-through, etc. The simplexmeshes used to build the DCS model present a number of useful features, e.g. simple implementation, fast computation, possibility of managing the surface and the centerline within the same formalism, easy control of the regularity of the surface.

The segmentation using the DSC model provides reasonably accurate measurements of the local diameters of the arterial lumen and of the length and curvatures of its centerline. These measurements are theoretically sufficient for the user to appropriately choose the dimensions of the stent, namely its length and its nominal diameters at the attachment sites. However, even a simple geometric simulation like ours makes this choice more intuitive, as each parameter of the stent can be interactively modified and the predictable result of 
this modification can be displayed online. Our geometric simulation tool is a step towards a complete pre-operative simulation of vascular stenting. Like other authors, it implicitly assumed that the axial rigidity of the arteries is much larger than the axial rigidity of the stents, while the radial rigidity of the stents is larger than that of the arteries. In other words, in our simulation, the axial shape of the stent is modified so that it fits to the centerline of the artery, while the arterial diameters between the attachment sites are modified so that they fit to the diameters of the deployed stent. In real situations however, the axial shape of the vessel may be modified by the stent, and the deployment of the stent may locally be limited by the rigidity of the vascular wall, e.g. owing to calcifications. In aneurysms, as the shape of the central part of the endoprosthesis is not constrained by the arterial wall, the centerlines of the artery and of the endo-prosthesis may differ significantly from each other. There are namely frequent problems with the angulation of the endo-prosthesis near the attachment sites in strongly curved vessels. A future version of our simulation tool should therefore involve a simulation of the local mechanical interaction between the stent and the vascular wall.

The usefulness of the extraction of the patient-specific vascular geometry and of a realistic simulation of the deployment of the stents may go beyond the pre-operative choice amongst the existing stents. These tools can namely be used for the design of patient-specific stents. Furthermore, the predictive value of the simulations is to be exploited to assess the outcome of the endo-vascular remodeling in terms of hemodynamics, as proposed by researchers from the Stanford University. To this purpose, the simplex mesh simulating the remodeled surface of the vascular lumen will be used to generate a volumetric finite-elements model exploitable in the computations of the fluid dynamics.

\section{CONCLUSION}

A surface model for vessel segmentation and representation was used, first, to extract a vascular segment of interest and, second, to simulate an interaction between the vessel intraluminal wall and a stent.

The accuracy of the intra-luminal surface reconstruction is beyond the sub-voxel resolution of the tested physical phantom images. In other words, we obtained a mean segmentation error lower than the spatial resolution (voxel size) of the images.

Moreover, the cylinder deformation model is simple and requires a short computation time, which is compatible with actual clinical procedures. The physician can interactively deploy the stent and assess the procedure: stent localization, final deployment parameters (radius and length) and a modified 3D surface showing the change in the vascular shape due to the presence of a deployed stent.

\section{ACKNOWLEDGEMENTS}

This work has been supported by Rhone-Alpes Region project ADeMo and by ECOS-Nord Committee (action C03S02). It falls within the scope of the scientific topics of GdR STIC-Santé. The authors are grateful to Professor
Philippe C. Douek, Dr Alejandro de la Vega and Dr JeanMichel Serfaty for providing MRA images and their medical expertise, and to Dr Marcela Hernández-Hoyos for her help in making links with Maracas software.

\section{REFERENCES}

[1] S. Thurnher and M. Grabenwöger, "Endovascular treatment of thoracic aortic aneurysms: a review," European Radiology, vol. 12, pp. 13701387,2002

[2] J.-M. Isokangas, R. Hietala, J. Perälä, and O. Tervonen, "Accuracy of computer-aided measurements in endovascular stent-graft planning,' Investigative Radiology, vol. 38, no. 3, pp. 164-170, 2003.

[3] K. Coenegrachts, H. Rigauts, and J. De Letter, "Prediction of aortoiliac stent graft length: comparison of a semiautomated computed tomography angiography method and calibrated aortography," Journal of Computer Assisted Tomography, vol. 27, no. 2, pp. 284-288, 2003.

[4] M. Orkisz and M. Hernández-Hoyos, "Models for 3d vascular image analysis," Journal of Medical Informatics and Technologies, vol. 2, no. 1, pp. IP13-IP22, 2001

[5] C. Taylor, M. Draney, J. Ku, D. Parker, B. Steele, K. Wang, and C. Zarins, "Predictive medicine: Computational techniques in therapeutic decision-making." Computer Aided Surgery, vol. 4, pp. 231-247, 1999.

[6] K. Wang, R. Dutton, and C. Taylor, "Improving geometric model construction for blood flow modeling," IEEE Engineering in Medicine and Biology, vol. 18, no. 6, pp. 33-39, 1999.

[7] N. Wilson, K. Wang, R. Dutton, and C. Taylor, "A Software Framework for Creating Patient Specific Geometric Models from Medical Imaging Data for Simulation Based Medical Planning of Vascular Surgery," in Medical Image Computer and Computer Assisted Intervention (MICCAI), ser. Lecture Notes in Computer Sciences, Utrecht, The Netherlands, 2001, pp. 449-456.

[8] J. Cebral, R. Löhner, O. Soto, P. Choyke, and P. Yim, "PatientSpecific Simulation of Carotid Artery Stenting Using Computational Fluid Dynamics," in Medical Image Computer and Computer Assisted Intervention (MICCAI), Utrecht, The Netherlands, 2001, pp. 153-160.

[9] P. Yim, J. Cebral, M. Rakesh, H. Marcos, and P. Choyke, "Vessel surface reconstruction with a tubular deformable model," IEEE Transactions on Medical Imaging, vol. 20, no. 12, pp. 1411-1421, 2001.

[10] C. Stern, S. Wildermuth, J. Weissmann, P. Stucki, P. Hilfiker, and J. Debatin, "Predictive medicine: Computational techniques in therapeutic decision-making." in Computer Assisted Radiology and Surgery (CARS), Paris, France, 1999, pp. 176-180.

[11] P. Yim, G. Vasbinder, V. Ho, and P. Choyke, "Isosurfaces as deformable models for magnetic resonance angiography," IEEE Trans. Med. Imaging, vol. 22, no. 7, pp. 875-881, 2003.

[12] G. Abdoulaev, S. Cadeddu, G. Delussu, M. Donizelli, L. Fromaggia, A. Gianchetti, E. Gobbetti, A. Leone, C. Manzi, P. Pili, A. Scheinine, M. Tuveri, A. Varone, A. Veneziani, G. Zanetti, and A. Zorcolo, "ViVa: The Virtual Vascular project," IEEE Transactions on Information Technology in Biomedicine, vol. 22, no. 4, pp. 268-274, December 1998.

[13] A. Zorcolo, E. Gobbetti, G. Zanetti, and M. Tuveri, "A Volumetric Virtual Environment for Catheter Insertion Simulation," in Eurograhics Workshop on Virtual Enviroments (EGVE), R. van Liere and J. Mulder, Eds. Amsterdam, The Netherlands: Springer-Verlag, June 2000.

[14] H. Delingette, "General Object Reconstruction based on Simplex Meshes," International Journal of Computer Vision, vol. 32, no. 2, pp. 111-146, 1999.

[15] P. Douek, D. Revel, S. Chazel, B. Falise, J. Villard, and M. Amiel, "Fast MR angiography of the aortoiliac arteries and arteries of the lower extremity: value of bolus-enhanced, whole-volume subtraction technique." American Journal of Radiology, vol. 165, pp. 431-437, 1995.

[16] M. Hernández-Hoyos, M. Orkisz, P. Puech, C. Mansard-Desbleds, P. Douek, and I. Magnin, "Computer-assisted analysis of threedimensional angiograms," RadioGraphics, vol. 22, pp. 421-436, 2002.

[17] B. Verdonck, J. Bloch, H. Maître, D. Vandermeulen, P. Suetens, and G. Marchal, "Blood vessel segmentation and visualization in 3D MRA and spiral CT angiography," in Computer Assisted Radiology (CAR). Berlin, Germany: Springer-Verlag, 1995, pp. 177-182.

[18] O. Wink, W. Niessen, and M. Viergever, "Fast delineation and visualization of vessels in 3d angiography images," IEEE Transactions on Medical Imaging, vol. 198, pp. 337-346, 2000.

[19] _ - "Minimum cost path determination using a simple heuristic function," in International Conference on Pattern Recognition (ICPR). Barcelona, Spain: IEEE Computer Society, 2000, pp. 1010-1013. 
[20] A. Frangi, W. Niessen, R. Hoogeveen, T. van Walsum, and M. Viergever, "Quantitation of vessel morphology from 3D MRA," in Medical Image Computer and Computer Assisted Intervention (MICCAI). Cambridge, UK: Springer-Verlag, 1998, pp. 358-367.

[21] N. Flasque, M. Desvignes, J.-M. Constans, and M. Revenu, "Accurate detection of 3D tubular tree structures," in International Conference on Image Processing (ICIP). Vancouver, Canada: Springer-Verlag Inc., 2000, pp. 436-439.

[22] K. Krissian, G. Malandain, N. Ayache, R. Vaillant, and Y. Trouuset, "Model based multiscale reconstruction of 3D vessels," in Computer Vision and Pattern Recognition (CVPR). Santa Barbara, CA , USA: IEEE, 1998, pp. 722-727.

[23] D. Paik, C. Beaulieu, R. Jeffrey, G. Rubin, and S. Napel, "Automated flight path planning for virtual endoscopy," Medical Physics, vol. 25, no. 5, pp. 629-637, 1999.

[24] S. Aylward and E. Bullitt, "Initialization, noise, singularities and scale in height ridge traversal for tubular object centerline extraction," IEEE Transactions on Medical Imaging, vol. 21, no. 2, pp. 61-75, 2002.

[25] C. Toumoulin, C. Bołdak, J.-L. Dillenseger, J.-L. Coatrieux, and Y. Rolland, "Fast detection and characterization of vessels in very large data sets using geometrical moments," IEEE Transactions on Biomedical Engineering, vol. 48, no. 5, pp. 604-606, 2001.

[26] S. Young, V. Pekar, and J. Weese, "Vessel segmentation for visualization of MRA with blood pool contrast agent," in Medical Image Computer and Computer Assisted Intervention (MICCAI), ser. Lecture Notes in Computer Sciences. Utrecht, The Netherlands: Springer Verlag, 2001, pp. 491-498.

[27] T. Deschamps and L. Cohen, "Fast extraction of minimal paths in 3d images and applications to virtual endoscopy," Medical Image Analysis, vol. 5, pp. 281-299, 2001.

[28] M. Orkisz and M. Hernández-Hoyos, "From inertia matrix to the analysis of vascular pathologies," in Int. Conf. Computer Vision and Graphics, vol. 1, Zakopane, Poland, 2002, pp. 6-15.

[29] T. McInerney and D. Terzopoulos, "Deformable models in medical image analysis : a survey," Medical Image Analysis, vol. 1, no. 2, pp. 91-108, 1996.

[30] J. Montagnat and H. Delingette, "Globally constrained deformable models for 3D object reconstruction," Signal Processing, vol. 71, no. 2, pp. 173-186, December 1998.

[31] M. Hernández-Hoyos, "Segmentation anisotrope 3d pour la quantification en imagerie vasculaire par résonace magnétique," Ph.D. dissertation, INSA de Lyon, France, July 2002.

[32] C. Renaudin, B. Barbier, R. Roriz, D. Revel, and M. Amiel, "Coronary arteries: new design for three-dimensional arterial phantom," Radiology, vol. 190, pp. 579-582, 1994.

[33] S. Shafer, Shadows and Silhouettes in Computer Vision. Boston: Kluwer Academic Publishers, 1985.

[34] E. Catmull and R. Rom, Computer Aided Geometric Design. New York, USA: Academic Press, 1974, ch. A Class of Local Interpolating Splines, pp. 317-336.

[35] C. Dumoulin and B. Cochelin, "Mechanical behaviour modelling of balloon-expandable stents," Journal of Biomechanics, vol. 33, pp. 14611470,2000

[36] A. Frangi, W. Niessen, R. Hoogeveen, T. Walsum, and M. Viergever, "Model-based quantitation of 3-d magnetic resonance angiographic images," IEEE Transactions on Medical Imaging, vol. 18, no. 10, pp. 946-956, 1999. 